\title{
Análisis del perfecto fuerte en cuatro fragmentos de obras medievales en castellano*
}

\author{
Patricia Fernández Martín ${ }^{* *}$ \\ Universidad Complutense de Madrid, España
}

\begin{abstract}
Resumen
En el presente artículo se proponen dos objetivos: i) comprobar el grado de frecuencia de aparición del perfecto fuerte en el período correspondiente en las obras estudiadas y ii) analizar si los verbos que en la actualidad siguen manteniendo las formas fuertes del perfecto son los mismos que aquellos que sufren una fuerte gramaticalización plausible en expresiones idiomáticas o perífrasis verbales. Para ello, se expondrán los resultados del análisis de los cuatro fragmentos de obras medievales y se llegará a la conclusión de que la frecuencia de uso de las formas de perfecto de determinados verbos se mantiene con el paso del tiempo a pesar de su aparente irregularidad. Esto implicará,
\end{abstract}

* Este trabajo se presentó en el I Congreso Internacional Las Lenguas y sus Literaturas organizado por la Asociación de Jóvenes Investigadores Filólogos (AJIF-UCM) de la Universidad Complutense de Madrid, celebrado los días 4, 5 y 6 de octubre de 2007. Asimismo, se enmarca dentro del Proyecto de Investigación FFI2008-02828/FILO financiado por el Ministerio de Ciencia e Innovación (España).

** Para correspondencia dirigirse a: Patricia Fernández Martín (patriciafernandezmartin@ gmail.com), Departamento de Lengua Española, Teoría de la Literatura y Literatura Comparada, Facultad de Filología, Universidad Complutense de Madrid, Edificio D, Avda. de la Complutense, s/n, 28040 - Madrid, España. 
finalmente, la hipótesis de que cuanto más se usa una palabra, menos probabilidades parece haber de que esa palabra cambie.

Palabras clave: perfecto fuerte o rizotónico, gramaticalización, robustez léxica.

\title{
Analysis of the STRONG fORMS IN Simple Past in fOUR fragments of Spanish Mediaeval Texts
}

\begin{abstract}
There aim of this paper is twofold: on the one hand, to determinate the frequency of Simple Past Tense forms in strong verbs is to analizes in written Spanish texts between XII and XIV century. On the other hand, to verify whether the verbs in Modern Spanish which keep on using strong forms in Simple Past Tense are the same verbs which undergo a strong process of gramaticalization in numerous idioms or verbal periphrasis. Thus, the main conclusion will take into account that the frequency of usage of the Simple Past Tense forms in some strong verbs remains the same in spite of being apparently irregular. This might allow us to think that the more frequently used a word is used, the fewer are the chances that that word changes.
\end{abstract}

Key words: strong verbs perfect, grammaticalization, lexical robustness.

Recibido: 31/03/09. Aceptado: 07/09/09.

\section{INTRODUCCIÓN}

En el presente trabajo, nos hemos propuesto dos objetivos que están interrelacionados: por un lado, comprobar el grado de frecuencia de aparición de las formas fuertes en el período correspondiente en cuatro fragmentos de obras medievales; y, por otro, analizar si los verbos que en la actualidad siguen manteniendo las formas fuertes del perfecto son los mismos que aquellos que sufren una fuerte gramaticalización en expresiones idiomáticas o perífrasis verbales (Luquet 1996; Bybee 1999). 
Para ello, vamos a exponer brevemente la evolución que sufrió la forma de pretérito perfecto ${ }^{1}$ desde el latín al español actual, y nos vamos a detener en un período de cuatro siglos (s. XII O XIII-XV), analizando cuatro fragmentos de obras pertenecientes a dicho período: el primer cantar del Poema de Mio Cid, compuesto, seguramente, a finales del XII o principios del s. XIII (a partir de ahora PMC); los capítulos 1-60 de la Primera Crónica General de España, perteneciente a mediados o finales del s. XIII (a partir de ahora $P C G$ ); el año onceno de la Crónica del rey don Pedro primero de finales del s. XIV (a partir de ahora, don Pedro); y los capítulos I-XV de La Ilíada de Homero de Juan de Mena, compuesta a principios del s. Xv (a partir de ahora, Ilíada).

La metodología seguida ha comenzado por la extracción de todas las formas del perfecto halladas y su consiguiente recuento, como se podrá comprobar en el apartado III.

Después, se han relacionado los datos extraídos con la evolución esperable, y se han establecido las conclusiones correspondientes.

\section{EVOLUCIÓN DEL PERFECTO}

\section{FoRMACIÓN DEL PRETÉRITO PERFECTO EN LATÍN}

En la lengua latina convivían diversas maneras de formar este tiempo verbal, como es sabido, partiendo siempre del tema de perfecto correspondiente a cada uno de los verbos.

Sintetizamos las principales a continuación (Beltrán 1999: 152-159; Lloyd 1993: 165-171).

En primer lugar, el perfecto se formaba en $-\mathrm{U}$ (llamado perfecto en $-\mathrm{U}$, -UI $\mathrm{o}-\mathrm{VI}$ ) en la mayoría de los verbos de las conjugaciones I, II y IV, cuyo radical terminaba en vocal. Es el caso de AMO - AMAUERUNT.

Desde muy temprano, en las formas del pretérito que se formaban así se va a producir la síncopa del grupo -ue- ante - $r$-, como en AMA(UE)RUNT;

Cuando en este trabajo utilizamos la palabra "perfecto", nos estamos refiriendo al tiempo verbal denominado "pretérito perfecto simple" (RAE 1973: § 2.11.1a; Alarcos 1980), también llamado por algunos autores "pretérito indefinido" (Alcina y Blecua 1994: § 5.2.3; Gómez Torrego 2002: 147, 150) o simplemente "pretérito" (Bello 1981: 349-355; 400-401). 
del grupo - $u i$ - ante $-s$, como sucede en AMA(UI)STI; y de la vocal media alta -u- entre vocales anteriores, como en AUDI(U)IT (Beltrán 1999: 154).

En segundo lugar, se encontraba el llamado perfecto sigmático o en -SI, que añadía a la raíz una sibilante, como en DICO - DIXI, UEHO - UEXI. Había algunas reglas que conviene matizar (Beltrán 1999: 154):

* Cuando se encontraba una dental precediendo al fonema /s/, el conjunto fonológico se transformaba en /si/, como en MITTO - MISI o RIDEO - RISI.

* Si la consonante que precedía a la sibilante era velar o aspirada, estábamos entonces ante $-x$ - (/ks/): IUNGO - IUXI; RĔGO - RĒXI; TRAHO - TRAXI; COQUO -COXI.

* Si la consonante anterior a /s/ tenía el rasgo [+ labial], entonces pasaba a ser /psi/, como en los verbos SCRIBO - SCRIPSI y SAEPIO - SAEPSI.

- Cuando la consonante antepuesta a la alveolar fricativa sorda oral era nasal, aparecía entonces un grupo consonántico compuesto por dicha nasal, una consonante epentética / $\mathrm{p} /$ y la sibilante correspondiente /s/, como en los ejemplos de DEMO - DEMPSI y PROMO - PROMPSI.

* Finalmente, cuando la raíz terminaba en /s/ se procedía a la simplificación (-sī) o al mantenimiento del grupo resultante (-SSİ): HAEREO - HAESI, HAURIO - HAUSI, para el primer fenómeno; GERO - GESSI; URO - ussi, para el segundo.

En tercer lugar, existía lo que se ha denominado perfecto alargado, en el que se producía un alargamiento de la vocal radical en el tema de perfecto: LĂVO - LĀVI.

Este cambio con respecto a la vocal radical del tema de presente podía darse en sentido estricto, manteniendo el timbre de la vocal y cambiando, por tanto, solamente su cantidad en el tema de perfecto (ĚDO - ËDI; FŬGIO - FŪGI; UĚNIO - UĒNI); o se podía dar, además, insertando una vocal de distinto timbre a la vocal radical del tema de presente (F $\breve{C} \mathrm{CIO}$ - FĒCI; $\breve{G O}$ - ĒGI) (Lloyd 1993: 167-168).

En cuarto lugar, existía otro perfecto que se formaba mediante el sistema fonológico de reduplicación (es el perfecto reduplicado). En este caso, se antepone una sílaba a la raíz, formada por la consonante inicial radical + una vocal, generalmente /e/. Solo se da en la III conjugación (PENDEO - PEPENDI; MORDEO - MOMORDI) y en la I (DO - DEDI; STO - STETI). No era productiva ya en el propio latín, debido a dos motivos: i) no se trata de una estructura morfológica demasiado transparente, puesto que la sílaba radical podía sufrir metafonía (CAEDO - CECĪDI); y ii) los verbos compuestos a partir de un verbo que sí reduplicaba en su forma perfecta no admiten este tipo de reduplicación (CURRO - CUCURRI, pero RECURRO - RECURRI). Por estos 
motivos acabó siendo reemplazado por el perfecto sin reduplicación de los compuestos (tipo RECURRI). De ahí que no dejara ningún resto en las lenguas romances (Beltrán, 1999: § 138, § 139, § 140).

Por último, había otra manera de formar el perfecto en latín, que era emplear el mismo tema para el presente y para el perfecto (éste ha sido denominado perfecto simple), y tomando las desinencias como únicas marcas diferenciales: BIBO - BIBI; SīDO - SĪDĪ; İCO -īCI (Beltrán 1999: § 143).

En términos generales, se podría decir que los perfectos débiles o sufijados vendrían, mayormente, del perfecto en $-\mathrm{U}$, del perfecto simple y de algunos alargados; y los perfectos fuertes o radicales se formarían siguiendo, en muchas ocasiones, la regla de los sigmáticos, los reduplicados y los demás alargados.

Por lo que respecta a las desinencias latinas ${ }^{2}$, había un grupo de desinencias para formar los perfectos. En su paso al protorromance, y al desaparecer la consonante labiodental de la I conjugación en todas las personas $([\mathrm{w}]>[\ldots])$ (Lloyd, 1993: 166), quedaron dos grupos posibles como modelos de desinencias de perfecto, el de la I y el de la IV, que eran, además, las más productivas:

\begin{tabular}{|c|c|c|c|c|c|c|}
\hline & $\mathbf{1}^{\mathrm{a}}$ & $2^{a}$ & $3^{\mathbf{a}}$ & $4^{a}$ & $5^{a}$ & $6^{a}$ \\
\hline I & $-\mathrm{ai}$ & -āstī & -āut & -ĀMUS & -ĀSTIS & -ĀRUNT \\
\hline IV & $-\mathrm{II}$ & -ĪSTĪ & -İUT & -ĪMUS & -İSTIS & -I(E)RUNT \\
\hline
\end{tabular}

Para comprender la evolución desde este paradigma al del español antiguo (o incluso al del español moderno), es necesario fijarse en la $3^{\mathrm{a}}$ persona, terminada en -ĀUT en los verbos de la I conjugación, y en -īUT en los de la IV, ya que parece ser la forma más transparente desde un punto de vista morfológico, puesto que está compuesta por la raíz + la vocal temática acentuada + desinencia $/-u t-/$. 


\section{Proceso evolutivo ${ }^{3}$}

En una primera fase inserta en el propio latín, la forma de construir el perfecto de los verbos en -UI se irá extendiendo a algunos verbos que no habían tenido esa forma de perfecto anteriormente. Por ejemplo, el verbo CADERE, que formaba el perfecto mediante la reduplicación (CECIDI) acabaría haciéndolo siguiendo las reglas del perfecto en - $u$ - (CADUĪ).

Sin embargo, la mayoría de las formas que presentaban reduplicación fueron sustituidas frecuentemente por perfectos sigmáticos (PEPIGI de PANGERE, pasa a ser PANXİ), mientras que verbos con perfectos construidos alargando la vocal radical desaparecieron completamente, y fueron sustituidos por otros que formaban el pretérito siguiendo el patrón regular de la I conjugación.

En una segunda fase, se va poco a poco instaurando la tendencia a seguir a los verbos de las I y IV conjugaciones latinas como modelos generalizados de perfecto, y se ve en la $1^{\mathrm{a}}$ persona una guía para todas las demás, especialmente de la $2^{\mathrm{a}}$ (del etimológico - aste se pasa al analógico - este, mucho más cercano del $-i$ de la $1^{\mathrm{a}}$ persona). También se da una influencia de la $2^{\mathrm{a}}$ persona sobre la $5^{\mathrm{a}}$, y entre ésta y la $4^{\mathrm{a}}$.

La $3^{\text {a }}$ persona, por su parte, toma definitivamente de las formas débiles la desinencia latina -AUT en verbos de la I conjugación, e -IUT en verbos de la IV.

Queda un grupo de verbos fuertes o rizotónicos que mantienen el modelo de la II conjugación, entre el cual se encuentra HABERE, al que toman como ejemplo verbos como JACUI, JACUISTI y PLACUI, PLACUISTI. SAPERE Y CAPERE rehacen su perfecto siguiendo el mencionado modelo en -UI y crean así un paradigma en español medieval hobo, sopo, copo que dará hubo, supo, сиро en español moderno.

También siguiendo al verbo HABERE otro grupo constituyó su perfecto: tener, tove; creer o crecer, crove; andar, andove; seer, sove; estar, estove.

Por lo que respecta a los perfectos sigmáticos, la mayor parte de ellos desaparece, aunque alguno se mantiene (como dixo en $P M C, P C G$; aduxo en $P C G$ o traxo en Ilíada) hasta la actualidad. Aparecen en formas compuestas perfectos que siguen el modelo sigmático, tales como requiso.

3 Para este apartado se han utilizado ejemplos de nuestro inventario, pero se ha recurrido a Lloyd (1993: 275-277, 478-492 y 579-584). La tabla-resumen del final de la sección ha sido elaborada por la autora del presente trabajo, teniendo en cuenta todos los datos hasta aquí expuestos. 
En una fase posterior, se van estabilizando las formas de las $2^{\mathrm{a}}, 4^{\mathrm{a}}$ y $5^{\mathrm{a}}$ personas, configuradas, seguramente, sobre la $1^{\text {a }}$; y la gran mayoría de los perfectos rizotónicos va cediendo en el uso a los arrizotónicos, que seguían un paradigma regular. Esto es lo que trataremos de comprobar en el análisis del corpus.

Para simplificar lo expuesto hasta aquí, presentamos a continuación una tabla en la que se trata de resumir el proceso evolutivo de las formas rizotónicas o fuertes.

\begin{tabular}{|c|c|c|}
\hline Latín clásico & Latín tardío & Español antiguo \\
\hline $\begin{array}{l}\text { Perfecto en -U, } \\
\text {-UI } 0-\text { VI }\end{array}$ & $\begin{array}{c}\cdot \text { Se extiende a distintos } \\
\text { tipos de verbos que no } \\
\text { hacían el perfecto en -U. } \\
\text { - Se mantiene como tal en } \\
\text { varios verbos de la II } \\
\text { conjugación. } \\
\text { Influencia del perfecto de } \\
\text { HABEO a verbos que no } \\
\text { hacían el perfecto en -UI. }\end{array}$ & $\begin{array}{c}\text {-Expansión de la } 1^{\mathrm{a}} \text { persona. } \\
\text { - Influencia de la } 2^{\mathrm{a}} \text { sobre la } 5^{\mathrm{a}} \text {. } \\
\text { - La desinencia de la } 3^{\mathrm{a}} \text { persona } \\
\text { es }-o<-\mathrm{UT}<-\mathrm{A} \mathrm{UT} / \text {-ĨUT } \\
\text { · Haber, hobo (ovo);saber, } \\
\text { sopo; caber, copo; tener, } \\
\text { tove; creer o crecer, crove; } \\
\text { andar, andove; seer, sove; } \\
\text { estar, estove }\end{array}$ \\
\hline $\begin{array}{l}\text { Perfecto } \\
\text { sigmático }\end{array}$ & & $\begin{array}{l}\text { La mayor parte de ellos } \\
\text { desaparece, excepto quiso, }\end{array}$ \\
\hline $\begin{array}{l}\text { Perfecto } \\
\text { reduplicado }\end{array}$ & Perfecto sigmático & $\begin{array}{c}\text { priso, miso, puso, remaso, } \\
\text { riso, escriso, cinxo, coxo, } \\
\text { destruxo, tinxo, yxo, dixo, } \\
\text { aduxo... } \\
\text {. Otro grupo se adhiere a } \\
\text { éste }^{4} \text { : fuxo, tanxo... }\end{array}$ \\
\hline $\begin{array}{l}\text { Perfecto } \\
\text { alargado }\end{array}$ & \multirow[t]{2}{*}{ Perfecto simple } & \multirow{2}{*}{$\begin{array}{l}\text { Se acaba creando paradigma } \\
\text { a partir de esta forma de } \\
\text { realizar el perfecto: } \\
\text { se regulariza (nivelación) }\end{array}$} \\
\hline Perfecto simple & & \\
\hline
\end{tabular}

En conclusión, en castellano medieval (y moderno) van a quedar dos grandes grupos de pretéritos: los perfectos rizotónicos o fuertes y los débiles $\mathrm{o}$ arrizotónicos. Los primeros son aquellos pretéritos que trasladan el acento

\footnotetext{
4 Según Paul M. Lloyd (1993: 489-490), habría que buscar el origen del par nasco - visco en la influencia francesa del siglo XI.
} 
de los morfemas gramaticales agudos al lexema, lo cual provoca, por un lado, una modificación en dicho lexema (querer $\rightarrow$ quise, y no *queri), y por otro, su combinación con una serie de desinencias exclusivas para este tiempo, que son, en términos generales, las derivadas de las incluidas anteriormente en el grupo I (Alcina y Blecua, 1995: 775; Lloyd 1993: 275). El pretérito débil o arrizotónico, por su parte, nunca lleva el acento en la raíz, sino en la desinencia (comí, be $\underline{b i}$ ), lo cual implica que va a mantener el radical del tema de presente, a diferencia de lo que ocurre con los rizotónicos que recurren al lexema del tema de perfecto, y además se combinan, en general, con las desinencias evolucionadas del grupo IV (Lloyd 1993: 275).

Dentro de los perfectos fuertes o rizotónicos nos encontramos en el español medieval con dos subgrupos: los pretéritos sigmáticos (aquellos que tienen un fonema sibilante en la raíz, como dixo, quiso o traxo) y aquellos que en latín formaban el perfecto con-u- (sopo, copo, ovo).

El resto de los verbos va a ir poco a poco acercándose al paradigma de los regulares, es decir, aquel perfecto denominado en la tabla anterior "perfecto simple".

\section{HIPÓTESIS INICIAL}

Resulta relativamente sencillo explicar por qué se va extendiendo la manera de realizar el perfecto débil latino a aquellas formas que en un primer momento lo hacían siguiendo patrones distintos: mediante un proceso morfológico de analogía, las formas del pretérito arrizotónico se iban expandiendo, en función de determinadas jerarquías (personas no marcadas frente a persona marcada; conjugaciones más o menos productivas) y siguiendo también determinadas reglas (por ejemplo, tendencia a no mantener la alomorfia cuando, además, una de las formas deja de ser transparente). La pregunta clave debería ser, entonces, por qué se mantienen las formas rizotónicas como tales, esto es, por qué ellas no se ven alcanzadas por la analogía.

Una cuestión que puede resultar interesante está relacionada con lo que se ha denominado redes semánticas o conexiones léxicas que, a grandes rasgos, establece que "cuando se adquieren palabras nuevas, se forman asociaciones con otras palabras que contienen los mismos rasgos" (Bybee 1999: 59). Uno de esos rasgos puede ser el nivel de frecuencia de uso en la lengua, de tal manera que, precisamente aquellas palabras con un nivel 
alto de frecuencia son más proclives a mantener la irregularidad que las caracteriza; por el contrario, palabras que son poco frecuentes acaban siendo empleadas siguiendo el patrón morfológico que siguen las palabras cuya frecuencia (y por tanto, productividad) es mayor, dada la robustez léxica según la cual parece funcionar nuestro lexicón. Dicha robustez léxica es la causante de que se creen las redes semánticas siguiendo un criterio de uso, y no estrictamente morfológico. Según esta hipótesis, cuanto más utilizado (más "robusto") sea un término, más probabilidades hay de que se mantenga inalterable a lo largo del tiempo (Bybee 1999) ${ }^{5}$.

De acuerdo con esto, se podría entender que, precisamente habría sido esa robustez léxica la que habría facilitado que determinados verbos con perfecto rizotónico se mantuvieran como tales en su paso a las lenguas romances: su accesibilidad en la mente de los hablantes sería mucho mayor, en general, que la de aquellos verbos cuya forma de perfecto era arrizotónica, y por tanto, regular, a pesar de que la frecuencia de uso de los primeros fuera inferior, en general, a la de los segundos. Este hecho en sí mismo no contradiría en absoluto la necesidad que habrían tenido los hablantes de recurrir al patrón morfológico más general cuando así lo hubieran visto necesario, dada su productividad (Bybee 1999: 73), esto es, cuando el verbo en cuestión hubiera disminuido su frecuencia de uso desde un punto de vista sociolingüístico: si un hablante se enfrenta a un verbo poco oído, las dudas sobre su formación morfológica serán mayores cuanto más desconocido sea dicho verbo, y por tanto, más probabilidad habrá de que tienda a formar sus tiempos verbales siguiendo el modelo común, según el principio de la analogía (Elvira 1998; Bybee 1999). Esa necesidad habría desaparecido en aquellos casos en que los verbos con perfecto rizotónico eran lo suficientemente constantes y frecuentes en el habla como para poder permitir, en la mente del hablante, el acceso a su forma irregular, sin necesidad de formarla o reflexionar sobre ella. Y, finalmente, parece que la gramaticalización es un factor más que puede influir en el hecho de que un verbo se convierta en un vocablo tremendamente frecuente en el habla (Luquet 1996).

Si todo esto mínimamente esbozado hasta aquí es cierto, lo esperable en nuestro corpus será encontrar, por un lado, un mantenimiento relativo en el empleo de formas del perfecto fuerte a lo largo de los siglos analizados, como corresponde a una frecuencia de uso constante; $y$, por otro lado, cierta regularidad en las entradas léxicas verbales que forman su perfecto partiendo

5 En Joan Bybee (1999: 61-62) se explica que son, precisamente, las personas $1^{\mathrm{a}}$ y $3^{\mathrm{a}}$ del singular las que más frecuencia de uso muestran. No puede ser casualidad, pues, que las formas rizotónicas afecten exclusivamente a estas dos personas. 
del patrón morfológico más irregular, como es de suponer, dada la naturaleza morfosintáctica-semántica de los verbos que así se comportan (Luquet 1996). En otras palabras: lo que podría aparecer en el análisis de nuestro inventario tiene que ver con que en cada obra exista más o menos la misma cantidad de formas fuertes, que las encontradas pertenezcan siempre a los mismos verbos de manera más estable y, quizá también que éstos se correspondan con aquellos en los que se produce gramaticalización.

\section{ANÁLISIS DEL CORPUS}

En este apartado, procederemos a analizar el corpus arriba mencionado, de manera progresiva: en primer lugar, nos centraremos en las formas halladas en $P M C$, especialmente en las formas fuertes; después, en las que pertenecen a $P G C$; más adelante, estudiaremos las encontradas en Don Pedro; y finalmente, estudiaremos las que estaban en Ilíada. Como antes hemos indicado, de cada una en las obras analizaremos la cantidad de perfectos fuertes existentes, así como cuáles son esos perfectos. De esta manera, realizaremos un estudio centrado únicamente en las formas rizotónicas medievales, pero desde una perspectiva tanto cualitativa como cuantitava.

Después, trataremos de establecer una relación cronológica del proceso en aquellos verbos mencionados en dichas obras para poder comprobar el desarrollo del proceso con datos fehacientes.

Antes de comenzar, sin embargo, vamos a mostrar una tabla en la que se observa el número de formas totales recogidas, sin contar las formas verbales que se derivan del pretérito perfecto:

\begin{tabular}{|c|c|c|c|c|}
\hline Obra & Verbos & Formas débiles & Formas fuertes & Formas totales \\
\hline PMC & 102 & 221 & 105 & 326 \\
\hline PCG & 204 & 1603 & 816 & 2419 \\
\hline Don Pedro & 80 & 275 & 223 & 498 \\
\hline Ilíada & 105 & 138 & 66 & 204 \\
\hline Total & 489 & 2234 & 1212 & 3447 \\
\hline
\end{tabular}

No hemos tenido en cuenta las formas derivadas; nos hemos centrado en las pertenecientes al perfecto, excepto en aquellos casos en los que hemos carecido de datos para juzgar la existencia de formas originales de pretérito, 
y hemos utilizado las derivadas correspondientes (pretérito imperfecto o futuro imperfecto de subjuntivo) para comprobar cómo sería el pretérito en esos verbos. Asimismo, hemos ejemplificado las formas verbales de otras personas distintas de la $1^{\mathrm{a}}$ y $3^{\mathrm{a}}$, cuando éstas no han constado en el corpus, para tratar de deducir a partir de ellas cuál sería la forma correspondiente, esto es, si se partiría del tema de presente o de perfecto. En el resto de los casos han sido completamente obviadas.

Hemos tenido en cuenta las formas en que se alternan los perfectos arrizotónicos con los perfectos rizotónicos, y la consiguiente desaparición (en obras posteriores) de uno de ellos. Se señalará en cada caso.

A la hora de hacer el recuento, los verbos dar, ver, ser e ir han sido considerados fuertes por ser rizotónicos, aunque somos conscientes de que lo ideal sería haberlos incluido dentro del grupo de los verbos mixtos, dado que es como realmente funcionan: acento en la raíz pero desinencias regulares (Elvira 1998: 126).

En cuanto a los verbos compuestos, han sido tenidos en cuenta como verbos diferentes a sus correspondientes originales a efectos de cómputo (avenir, requerir), excepto en aquellos casos cuya variación es estrictamente fonética o fonológica, y no llega a ser semántica (y mucho menos, morfológica), como por ejemplo crecer / acrecer; troverse / atroverse. No obstante, en el anexo incluido al final del trabajo se tienen en cuenta en función de la raíz verbal de la que provengan y su consiguiente comportamiento morfológico.

Asimismo, han sido incluidas absolutamente todas las formas encontradas, independientemente del contexto sintáctico en que aparezcan; esto implica que verbos insertos en perífrasis (tovo de ser), en formas verbales (ovo fecho) o en construcciones pasivas (fueron muertos) han sido estudiados en este trabajo ${ }^{6}$.

\section{DESCRIPCIÓN DE LOS DATOS ENCONTRADOS EN CADA OBRA}

\section{Poema de Mio Cid}

Esta obra, como ha podido comprobarse en la tabla anterior, contiene un $32,2 \%$ de formas fuertes con respecto a las formas totales encontradas en el fragmento analizado.

\footnotetext{
6 Como ya hemos indicado, al final del estudio se ha incluído un anexo en el que se puede ver cierta evolución en las formas del perfecto fuerte aparecidas en las obras mencionadas.
} 
Estas formas fuertes son las siguientes (se indica entre paréntesis cuántas se han encontrado en cada caso $)^{7}$ : cinxo (4); crovo (1); dio (6); dixo (16); fizo (7); fue (11); ovo (7); poso (2); priso (4); plogo (6); pudo (1); quiso (2); respuso (3); retovo (1); sonrriso (2); sopo (1); sovo (1); tovo (2); vio (8); vino (6); yogo (1).

Existe tan solo una alternancia entre el fuerte nasco (13 casos) y su correspondiente débil naçió (1 caso).

De todos los verbos aquí expuestos, los ejemplos de cinxo, priso, plogo, sonriso, nasco y yogo, aunque es sabido que no van a sobrevivir muchos siglos más (y muchos menos llegarán a nuestros días), aparecen, sin embargo, con una forma relativamente etimológica (cinxo procede de CINGO que tenía un perfecto sigmático CINXIT; la desinencia varió, como se ha dicho, adaptando la de la I conjugación terminada en -ĀUT, de una forma semejante a lo que le ocurrió a priso < PRENDIT, y a sonriso < (SON)RISIT; plogo $<$ PLACUIT y yogo $<$ JACUIT) en la que se mantiene la forma fuerte procedente ya del mismo latín.

El resto de los verbos mantendrán la forma fuerte hasta el español actual (excepto en el caso de sovo, que acabaría siendo desplazado por fue).

Dado que no hemos encontrado ningún ejemplo de verbos como andar o vivir recurrimos a las formas que pueden hacernos ver cómo sería ese perfecto simple en las $1^{\text {a }}$ y $3^{\mathrm{a}}$ personas, esto es, según los ejemplos de andidieron (2) o andidiste (1) y de visquiéredes (1), porque en obras posteriores sí aparecerán. Según estos casos, las formas correspondientes en la $1^{\mathrm{a}}$ y $3^{\mathrm{a}}$ personas serían rizotónicas.

\section{Primera Crónica General}

En esta obra, es el 33,7\% el que pertenece a las formas fuertes. Se han incluido tanto las pertenecientes a la primera persona como las de la tercera. Son las siguientes (el número entre paréntesis indica cuántos ejemplos hemos encontrado de esa forma): aduxe (1), aduxo (6); andut (1), andudo (5); avino (7); compuso (1); di (2), dio (18); dixo (53); estudo (5); fize (8), fizo (100); fue (226); fuxo (14); mantovo (1); priso (2); pudo (12); quise (1), quiso (26); ove (1), ovo (144); plogo (21); poso (2), puso (35); re(s)puso (3); sopo (26); sovo (2); tove (1), tovo (22); troxe (1), troxo (3); vi (2); vine (1); vino (22); visco (1).

${ }^{7}$ Los verbos ser e ir han sido computados como dos verbos diferentes, pero hemos unificado sus formas porque, desde un punto de vista morfológico, son idénticas. 
Hemos encontrado dos verbos alternantes: destruyó (5 casos) frente a destruxo (1 caso); y vio (39 casos) frente a vido (1 caso); y uno que seguramente lo fuera: crecieron (2 ejemplos) frente a crovieron (1).

Resulta asimismo llamativo que algunos compuestos formados a partir de verbos que hacen su perfecto siguiendo las reglas del fuerte, sean arrizotónicos. Es el caso de querer - quiso (26 ejemplos) frente a conquerir - conquirió (2 ejemplos). Y sin embargo, nos encontramos también verbos (ya mencionados) que se comportan de igual manera sea en su forma base o en su forma derivada: mantener - tener.

Dado que en el $P M C$ aparece la forma crovo (de creer o crecer), que no aparece en $P C G$, hemos recurrido a crovieron para comprobar cómo podría ser la forma correspondiente de la $3^{a}$ persona; y puesto que también hemos encontrado dos ejemplos de crecieron, podemos concluir, en principio, que ya habría empezado la confusión.

\section{Crónica del Rey Don Pedro}

El $44,7 \%$ de las formas totales recogidas del fragmento analizado de la obra en cuestión está compuesto por formas que siguen el patrón rizotónico. Los verbos que aparecen siguiendo el esquema del perfecto fuerte en esta obra son los siguientes: andudo (2); dio (4); detovo (1); dixo (33); estudo (6); fize (1), fizo (33); fue (63); fuxo (2); ovo (18); plogo (7); poso (1), puso (3); pudo (5); quiso (5); sopo (19); tovo (3); traxo (3); vino (4); vio (7).

Merece la pena destacar la alternancia del verbo prender, cuyo perfecto débil aparece en dos ocasiones (prendió), frente a una única en que aparece el perfecto fuerte (priso). Asimismo, la forma de la $6^{\text {a }}$ persona aparece siguiendo el patrón del tema de presente: prendieron (2 ejemplos).

Encontramos también un ejemplo de un verbo que forma el perfecto a la manera regular, y que resulta ser derivado de otro verbo cuyo perfecto es siempre fuerte: de requerir encontramos requirió (1) frente al quiso de querer.

Asimismo, merece la pena destacar la forma fuxo (2), ya mencionada, frente a la forma alternante de otras personas, pues, si bien hay dos casos de fuxeron que confirmarían la toma de la raíz desde el tema de perfecto, se han hallado también dos casos de fuyeron, que podrían dar una pista acerca de la posible vacilación que empezaría a sufrir este verbo durante esta época.

También nos hemos fijado en la forma visquió ( 2 ejemplos), porque si la ortografía no engaña y el grupo consonántico quió equivale a /kió/, esta palabra se habría formado de manera mixta, partiendo del tema de perfecto, presente en visco ( $3^{\mathrm{a}}$ persona), del cual no hemos encontrado ejemplos en esta parte del corpus, pero utilizando, sin embargo, las desinencias típicas 
de las formas características del perfecto débil. Sería otra manera de dejar constancia de la vacilación que empezaba a sufrir este verbo y, seguramente, de la que se empezaba a sentir en todas las formas verbales cuya frecuencia de uso hubiera disminuido considerablemente.

Por último, se debe resaltar el caso de truxieron porque, aunque pertenezca a una de las personas (la $6^{\mathrm{a}}$ ) en las que nunca va a ser posible la forma fuerte, es interesante comprobar que existe una vacilación entre la forma con vocal radical alta posterior y la palabra con vocal radical baja, constante en la $3^{\mathrm{a}}$ persona.

\section{La Ilíada de Homero}

En esta obra, el $32 \%$ de las formas totales sigue el patrón del perfecto fuerte. Son las siguientes: conduxo (1); detovo (1); dio (2); dixo (8); estuvo (1); fui (1), fue (18); fizo (5); ovo (4); plogo (1), plugo (1); propuso (1); supo (1); tovo (1); traxo (5); vi (1); vine (1), vino (10).

Solamente hemos encontrado un caso de alternancia: vido (4 ejemplos) y vio (1 ejemplo). Dado el carácter latinizante del estilo de Juan de Mena, cabe preguntarse si realmente este vido (aunque más frecuente que vio) sería lo suficientemente constante en la lengua hablada del momento como para poder aparecer en una obra del tipo que Juan de Mena está traduciendo, o si él recurre a esta forma verbal por cuestiones estrictamente estilísticas. Asimismo, es necesario matizar que la única forma vio que aparece en el texto está muy cercana a una de vido, y quizá este sea el motivo por el que utilice el traductor una variante del mismo tiempo verbal. En cualquier caso, es el único ejemplo de forma alternante que encontramos en este texto.

De manera indirecta podemos deducir cómo funcionaban algunos verbos cuya $3^{\mathrm{a}}$ persona del pretérito perfecto no hemos encontrado en nuestro corpus, ya que tenemos una forma derivada del verbo yacer (yacieres, $2^{\mathrm{a}}$ persona del singular del futuro de subjuntivo) y foir (fuyera, $3^{\mathrm{a}}$ persona del pretérito imperfecto de subjuntivo): parece que en ambos casos la forma fuerte había desaparecido por completo.

\section{IMPLICACIONES DE LOS DATOS}

Como ha podido observarse en el apartado anterior, los verbos que aparecen en el fragmento del $P M C$ analizado son prácticamente los mismos que aparecen en el resto de las obras: cinxo; crovo; dio; dixo; fizo; fue; ovo; poso; priso; plogo; pudo; quiso; respuso; retovo; sonrriso; sopo; sovo; tovo; vio; vino; yogo. 
Excepto en el caso de ceñir, que no vuelve a encontrarse, todos los verbos expuestos aparecen, frecuentemente, en la $P C G$ : di, dio; dixo; fize, fizo; fue; ove, ovo; plogo; poso, puso; priso; pudo; quise, quiso; re(s)puso; sopo; sovo; tove, tovo; vi; vine; vino.

A esta lista se le añaden verbos nuevos que construyen su perfecto siguiendo el modelo fuerte: aduxe, aduxo ( $<$ ADDŪXIT); andut, andudo; avino $(<$ (A)VENIT; se cierra la vocal radical por metafonía); compuso; estudo; fuxo; mantovo; troxe, troxo; visco.

Por lo que respecta a los verbos creer/crecer y yacer que forman el perfecto siguiendo el modelo fuerte en el $P M C$, resulta llamativo que ya en $P C G$ aparece la forma débil crecieron que puede indicar cómo se formaba el resto del paradigma; $y$, aunque sea inserto en una variante (aparentemente, fonológica) del verbo en cuestión, hemos encontrado también la $3^{a}$ persona acreció. Por lo que respecta al verbo yacer, y a pesar de que nuestro corpus carece de alguna otra forma de dicho verbo hasta la Ilíada, se puede imaginar que por las fechas de composición de la $P G C$ ya podría haber comenzado la vacilación, dado que se da también con el mencionado par crecieron/ crovieron, y destruyó/destruxo. Asimismo, hay que tener en cuenta, como ya se ha indicado, que verbos compuestos a partir de aquellos que construyen su perfecto siguiendo el modelo fuerte (como requerir) ya no lo utilizan: recurren al perfecto analógico del resto de verbos.

A los verbos que aparecen en $P M C$ y $P C G$ se les puede añadir tan solo uno hallado en el fragmento analizado de Don Pedro: detovo, que en realidad, puede ser considerado un compuesto a partir de tener, y por tanto, podría extrañar que hiciera su perfecto siguiendo ese modelo, a diferencia de lo que ocurre con requerir en Don Pedro o conquerir en $P C G^{8}$.

Como se ha indicado en su momento, resulta especialmente llamativa la palabra visquió, encontrada en esta obra, porque, de corresponderse a ciencia cierta la ortografía con la pronunciación [biskjó], estaríamos ante una forma mixta, puesto que se habría tomado el tema de perfecto (procedente de visco) pero las desinencias del paradigma regular. Esta forma se encuentra a caballo, pues, entre el visquiéredes (que permite deducir un visco) del $P M C$ y el visco propiamente dicho de la $P C G$, por un lado, y el posterior y

8 En el libro de Lloyd (1993: 486) se afirma que conquerir < CONQUĪRERE y requerir $<$ REQUIRERE llegaron a formar su perfecto siguiendo el modelo de querer, que pertenecía al grupo de verbos que tenía perfecto sigmático (quiso). Sin duda, tuvo que ser anterior a lo que hemos encontrado en nuestro corpus (o en algún fragmento que no se ha analizado), porque todos los casos referentes a estos verbos siguen las pautas del perfecto débil, como ya se ha señalado. 
definitivo vivió que podemos encontrar en la actualidad (y suponer a partir de ese visquió), por otro.

Por lo que respecta al conjunto de verbos encontrados en la Ilíada traducida por Juan de Mena, llama la atención conduxo, porque no ha aparecido como tal en otros fragmentos del corpus, aunque sigue la construcción del perfecto fuerte esperable, que es la que se encuentra en la raíz - ducir ( $<$ DUCERE); así como estuvo, que parece que ya ha abandonado la forma etimológica y ha adquirido la analógica, más semejante a hubo, supo, cupo, etc. Los verbos restantes son los mismos que hemos encontrado en obras anteriores.

Recopilando, pues, todos los verbos que en la Ilíada (nuestra última obra) siguen empleando el pretérito fuerte (conduxo, (de)tovo, dio, dixo, fue, fizo, ovo, plugo, supo, traxo, vino) y añadiéndoles aquellos que pertenecen a los fragmentos analizados de las obras anteriores (andudo, puso, pudo, quiso, tobo, vio, estuvo) obtenemos los mismos que siguen ese patrón en la actualidad: andar, dar, decir, -ducir, estar, haber, hacer, ir, poder, poner, querer, saber, ser, tener, traer, venir y ver ${ }^{9}$.

Ahora, pasamos a analizar el motivo por el que estos verbos se han mantenido construyendo un perfecto fuerte, y no ha sido así en otros (cinxo, yogo) (Luquet 1996: 406-408). Partiendo de la hipótesis que aquí planteamos, el motivo está relacionado con el significado semántico que aportan, pero también con su significado gramatical, esto es, con su permanencia en diferentes estructuras sintácticas que provocan una mayor frecuencia en esos verbos, y por tanto, una mayor relación entre sus formas irregulares y el acceso a esas formas.

Desde un punto de vista estrictamente semántico, estos verbos denotan conceptos básicos de la expresión humana (aquí puede estar la razón de ser de su robustez léxica): la existencia (ser, estar, haber), la posesión (haber, tener), la modalidad o probabilidad (querer, poder, saber, caber), el acto del lenguaje en sí mismo (decir), la actuación por antonomasia (hacer) y el movimiento (ir, venir).

Desde el punto de vista gramatical, es fácil tener en cuenta que los verbos más utilizados (ser, estar, haber) son también los que tienden

9 Son los verbos que se mantienen en la actualidad según Elvira (1998: 126). Hemos añadido ser a la lista. Y, según lo que hemos obtenido del corpus, en esa lista no se puede incluir el verbo placer porque en la actualidad apenas se usa en contextos literarios. Asimismo, falta el verbo caber del cual no hemos encontrado ningún ejemplo en nuestro inventario. Finalmente, hay que tener en cuenta que en esta obra los verbos aparecen sin compuestos, y en nuestro corpus cada verbo ha contado como uno, independientemente de su base léxica (excepto en los casos ya comentados, véase más arriba). 
a utilizarse como auxiliares en nuestra lengua (voz pasiva, estructuras resultativas, perífrasis perfectivas); con estos verbos se relacionan andar e ir que funcionan de manera semejante en muchas perífrasis verbales (andar preocupado, ir a bailar, ir anocheciendo). El verbo dar es base de una serie de formaciones léxicas en las que se ha gramaticalizado, esto es, ha perdido su significado más literal y ha adoptado uno figurado (dar un grito, dar un paseo). Finalmente, los verbos traer, venir, poner y la serie -ducir son bases de derivación de bastantes verbos españoles (lo cual implica que, conceptualmente, se anteponen a sus derivados) ${ }^{10}$.

Para concluir esta sección, ofrecemos una tabla en la que aparecen los verbos que forman su perfecto siguiendo un modelo denominado fuerte, junto con la cantidad total de verbos de cada obra y su respectivo porcentaje, para facilitar la comparación (se excluyen aquellos verbos con alternancia):

\begin{tabular}{|c|c|c|c|}
\hline Obra & $\mathbf{N}^{\mathbf{o}}$ verbos & $\begin{array}{c}\text { Verbos con } \\
\text { perfecto fuerte }\end{array}$ & Porcentaje \\
\hline$P M C$ & 102 & 21 & $20,5 \%$ \\
\hline$P C G$ & 204 & 25 & $12,25 \%$ \\
\hline Don Pedro & 80 & 19 & $23,75 \%$ \\
\hline Ilíada & 105 & 14 & $13,3 \%$ \\
\hline
\end{tabular}

Si ahora comparamos el porcentaje de verbos que forman el perfecto fuerte en nuestro corpus, con el porcentaje, también en cada obra, de formas construidas según este modelo, se puede llegar a la conclusión de que el porcentaje de uso de las formas verbales que emplean un modelo de perfecto fuerte es siempre superior al porcentaje de verbos que cumplen esta característica:

\begin{tabular}{|c|c|c|}
\hline Obra & $\begin{array}{c}\text { Porcentaje de } \\
\text { verbos con perfecto fuerte }\end{array}$ & $\begin{array}{c}\text { Porcentaje de formas } \\
\text { en perfecto fuerte }\end{array}$ \\
\hline$P M C$ & $20,5 \%$ & $32,2 \%$ \\
\hline$P C G$ & $12,25 \%$ & $33,73 \%$ \\
\hline Don Pedro & $23,75 \%$ & $44,7 \%$ \\
\hline Ilíada & $13,3 \%$ & $32 \%$ \\
\hline
\end{tabular}

10 En el caso de los verbos de la serie -ducir, resulta interesante que hoy en día cada vez son más los ejemplos que podemos encontrar en la lengua hablada de formaciones del perfecto de estos verbos sobre la base del perfecto débil (condució, sedució), lo cual puede implicar el comienzo del proceso de analogía de este grupo de verbos hacia los estrictamente regulares y su consiguiente alejamiento del paradigma de formación de perfectos fuertes. 
Según nuestro estudio, los verbos que emplean un perfecto fuerte son muy pocos comparados con los que construyen su perfecto en torno al patrón más regular; sin embargo, las formas verbales que siguen este modelo son muchísimo más frecuentes en comparación con la cantidad de verbos que tienen un perfecto fuerte (de 21 en el PMC a 14 en la Ilíada). La progresiva reducción, por otro lado esperable, de la cantidad de verbos con perfecto fuerte en nuestro inventario parece contradecirse con los ejemplos de $P C G$, donde debería encontrarse un número inferior a 21 , que es lo que aparece en $P M C$. No obstante, esta variación puede explicarse sobre la base de la cantidad de verbos existentes en uno y otro fragmento (apenas hay un punto de diferencia en el porcentaje correspondiente).

Así pues, se puede concluir de este breve análisis que la amplia frecuencia de uso de las formas verbales de tipo fuerte (procedentes, principalmente, de los perfectos latinos en -U, -UI o-VI y del sigmático), junto con la facilidad de esos verbos para ser empleados en estructuras gramaticales "cerradas" (es decir, en estructuras gramaticalizadas) y su inmediato empleo para expresar conceptos cognitivos fundamentales de la comunicación humana, han facilitado su permanencia durante tantos siglos dentro del paradigma correspondiente a los verbos que tienen un pretérito perfecto simple rizotónico en la actualidad.

\section{CONCLUSIONES}

En este breve análisis hemos comprobado que existe una clara evolución tanto cualitativa como cuantitativa en la formación del perfecto fuerte de los verbos castellanos.

Esta evolución, como se ha visto, afecta tanto a la cantidad de verbos que formaban su perfecto siguiendo los patrones del perfecto rizotónico, que tiende a ser menor, como a la cantidad de formas que se desprende de cada uno (o de todos) de esos verbos, que suele ser constante a lo largo de los siglos. La principal conclusión es, pues, que la frecuencia de uso de las formas de perfecto de determinados verbos se mantiene con el paso del tiempo (y de ahí su robustez léxica), y siempre se trata, al final, de los mismos lexemas verbales.

Esta misma idea se puede relacionar con el grado de accesibilidad que el hablante tiene, dentro de su lexicón, a cada una de las formas verbales (tema de presente frente a tema de perfecto, por ejemplo), es decir, la mayor o menor 
facilidad con que el hablante escoge determinada palabra dentro de todas las opciones posibles con las que cuenta. Esta accesibilidad, según hemos visto, habría sido constante con determinados verbos (los que mantienen un significado que hemos denominado básico, que son los que se transmiten a través de construcciones sintácticas mayor o menormente fijadas, esto es, más o menos gramaticalizadas) y muy inferior con aquellos otros que se alejan de lo más diariamente frecuente para volver a surgir, tiempo después, convertidos en formas del paradigma más común que es al que los acaba atrayendo la analogía.

Evidentemente, esta relación establecida a tres niveles (la frecuencia de las formas de perfecto fuerte, el porcentaje de verbos que escogen el pretérito rizotónico y el posible grado de gramaticalización de estos verbos en otras expresiones del mismo sistema lingüístico, como perífrasis, auxiliares, etc.) no es más que una hipótesis de trabajo que habría que contrastar con los datos encontrados en un corpus mayor, que preferentemente estuviera formado por diferentes tradiciones discursivas, perteneciera a diversas zonas geográficas y comprendiera un período cronológico más amplio.

Asimismo, sería muy interesante añadir a dicho análisis la relación entre las formas básicas (pretérito perfecto simple) y las formas derivadas del tema de perfecto (pretérito imperfecto y futuro simple, ambos de subjuntivo) con aquellas formadas (tanto originales como derivadas) a partir del tema de presente, para comprobar en qué medida habría ejemplos en los que una forma derivada se formara a partir del tema de presente y no del tema de perfecto, o a la inversa.

Por último, también sería deseable comparar todos estos datos con los porcentajes de uso de la forma de perfecto débil y el estudio de los verbos que han comenzado fluctuando entre la robustez de los verbos irregulares y el paradigma de los regulares y han terminado asentándose en uno de los dos grupos, y si el proceso de gramaticalización ha tenido algo que ver en ello. 


\begin{abstract}
ANEXO
En este anexo reunimos la evolución de los verbos que alguna vez han aparecido en perfecto fuerte. Unificamos las formas de los verbos compuestos para ilustrar el proceso evolutivo desde un punto de vista estrictamente formal. En aquellos casos en que haya dos entradas del mismo verbo en una misma celda, una se corresponderá con la $1^{a}$ persona y la otra con la $3^{\text {a }}$. Si se trata de distintos verbos, uno se encontrará en la forma básica y el otro con el verbo compuesto correspondiente. Cuando carecemos de datos de formas verbales en perfecto de manera explícita, utilizamos las formas derivadas (futuro o pretérito imperfecto de subjuntivo) como posibles muestras del funcionamiento esperable del perfecto del cual se derivan, o las formas de otras personas, si éstas nos pueden ayudar a saber cómo eran las fuertes. En este caso, se encuentran en versalita. Además, hemos puesto en negrita el infinitivo de aquellos verbos que mantienen la alternancia de las dos formas dentro de la misma obra; mantenemos en subrayado las formas verbales que son débiles, cuando se han dado en el mismo corpus pero en otro siglo formas de ese mismo verbo con el perfecto fuerte. Finalmente, en cursiva se hallan aquellos verbos que cabe esperar que dejen de formar su perfecto siguiendo el patrón rizotónico.
\end{abstract}




\begin{tabular}{|c|c|c|c|c|}
\hline Verbo & PMC & PCG & Don Pedro & Ilíada \\
\hline -duzir & & $\begin{array}{l}\text { aduxe } \\
\text { aduxo }\end{array}$ & & conduxo \\
\hline aver & ovo & $\begin{array}{l}\text { ove } \\
\text { ovo }\end{array}$ & ovo & ovo \\
\hline andar & ANDIDIERON & $\begin{array}{c}\text { andut } \\
\text { andudo }\end{array}$ & andudo & \\
\hline ceñir & cinxo & & & \\
\hline creer / crecer & crovo & $\begin{array}{c}\text { CROVIERON } \\
\text { CRECIERON } \\
\text { acreció }\end{array}$ & cresçió & $\underline{\text { creçió }}$ \\
\hline dar & dio & $\begin{array}{c}\text { di } \\
\text { dio }\end{array}$ & dio & dio \\
\hline destroír & & $\begin{array}{l}\text { destruyó } \\
\text { destruxo }\end{array}$ & & \\
\hline dezir & dixo & dixo & dixo & dixo \\
\hline estar & & estudo & estudo & estuvo \\
\hline fazer & fizo & $\begin{array}{l}\text { fize } \\
\text { fizo }\end{array}$ & $\begin{array}{l}\text { fize } \\
\text { fizo }\end{array}$ & fizo \\
\hline foír & & fuxo & $\begin{array}{c}\text { fuxo / FUXERON } \\
\text { FUYERON }\end{array}$ & FUYERA \\
\hline nascer & $\begin{array}{l}\text { nasco } \\
\text { nació }\end{array}$ & & nasçistes & \\
\hline plogar & plogo & plogo & plogo & plogo / plugo \\
\hline poner & poso & $\begin{array}{l}\text { poso / puso } \\
\text { compuso }\end{array}$ & poso / puso & propuso \\
\hline prender & priso & priso & prendió / priso & \\
\hline poder & pudo & pudo & pudo & \\
\hline querer & quiso & $\begin{array}{c}\text { quise } \\
\text { quiso } \\
\text { conquirió }\end{array}$ & $\begin{array}{l}\text { quiso } \\
\text { requirió }\end{array}$ & \\
\hline responder & respuso & re(s)puso & & \\
\hline saber & sopo & sopo & sopo & supo \\
\hline seer & sovo / fue & sovo / fue & \multirow{2}{*}{ fue } & fui \\
\hline ir & fue & fue & & fue \\
\hline sonreír & sonrriso & & & \\
\hline
\end{tabular}




\begin{tabular}{|c|c|c|c|c|}
\hline tener & $\begin{array}{c}\text { tovo } \\
\text { retovo }\end{array}$ & $\begin{array}{c}\text { tove } \\
\text { tovo } \\
\text { mantovo }\end{array}$ & $\begin{array}{c}\text { tovo } \\
\text { detovo }\end{array}$ & $\begin{array}{c}\text { tovo } \\
\text { detovo }\end{array}$ \\
\hline traer & troxe \\
troxo & $\begin{array}{c}\text { traxo } \\
\text { truxieron }\end{array}$ & traxo \\
\hline veer & vio & $\begin{array}{c}\text { vio } \\
\text { vido }\end{array}$ & vio & $\begin{array}{c}\text { vi } \\
\text { vio } \\
\text { vido }\end{array}$ \\
\hline venir & vino & $\begin{array}{c}\text { vine } \\
\text { vino } \\
\text { avino }\end{array}$ & vino & $\begin{array}{c}\text { vine } \\
\text { vino }\end{array}$ \\
\hline yazer & visQUIÉREDES & visco & visquió & \\
\hline
\end{tabular}




\section{REFERENCIAS BIBLIOGRÁFICAS}

Alarcos Llorach, Emilio. 1980. Perfecto simple y compuesto. En Estudios de gramática funcional del español. Madrid: Gredos, pp. 13-49.

Alcina Franch, Juan y José Manuel Blecua. 1994. Gramática española. Barcelona: Ariel.

Bello, Andrés. 1981. Gramática de la lengua castellana destinada al uso de los americanos, ed. Ramón Trujillo. Tenerife: Cabildo insular.

Beltrán, José A. 1999. Introducción a la morfología latina. Zaragoza: Universidad de Zaragoza.

Bybee, Joan. 1999. Modelo de redes en morfología, en Samper Padilla et al. (eds.). Actas del XI Congreso Internacional de la ALFAL. Las Palmas de Gran Canaria: Universidad, vol. 1, pp. 59-74

ElviRA, JAVIER. 1998. El cambio analógico. Madrid: Gredos.

Gómez Torrego, Leonardo. 2002. Gramática didáctica del español. Madrid: SM.

Lloyd, PAUl M. 1993. Del latín al español. I. Fonología y morfología históricas de la lengua española. Madrid: Gredos.

LuQuet, Gillet. 1996. Un caso de motivación del signo lingüístico: la oposición regular/ irregular en la historia de los pretéritos indefinidos, en Actas del III Congreso Internacional de Historia de la Lengua Española, Madrid: Arco/Libros, pp. 403-410.

Real Academia Española. 1973. Esbozo de una nueva gramática de la lengua española, Madrid: Espasa-Calpe.

\section{CORPUS}

Alfonso X el Sabio (ed. Menéndez Pidal). 1977. Primera Crónica General de España. Madrid: Gredos.

López de Ayala, P. (ed. Germán Orduna). 1994. Crónica del rey don Pedro y del rey don Enrique, su hermano, hijos del rey don Alfonso Onceno. Buenos Aires: Secrit.

Mena, JuAn (eds. González Rolán, T; López Fonseca, A.; Barrio Vega, Ma F.). 1996. La Ilíada de Homero. Madrid: Ediciones Clásicas.

Poema de Mio Cid (ed. Colin Smith). 1998. Madrid: Cátedra. 